\title{
PROJECTIVITY OF A RELATIVE HOPF MODULE OVER THE SUBRING OF COINVARIANTS
}

\author{
S. CAENEPEEL AND T. GUÉDÉNON
}

\begin{abstract}
Let $k$ be a commutative ring, $H$ a faithfully flat Hopf algebra with bijective antipode, $A$ a $k$-flat right $H$-comodule algebra. We investigate when a relative Hopf module is projective over the subring of coinvariants $B=A^{\mathrm{co} H}$, and we study the semisimplicity of the category of relative Hopf modules.
\end{abstract}

\section{INTRODUCTION}

Let $H$ be a Hopf algebra with bijective antipode, and $A$ a left $H$ module algebra. We can then form the smash product $A \# H$. In [8], the second author investigated necessary and sufficient conditions for the projectivity of an $A \# H$-module $M$ over the subring of invariants $A^{H}$.

Our starting point is the following: if $H$ is finitely generated and projective, then $H^{*}$ is also a Hopf algebra, $A$ is a right $H^{*}$-comodule algebra, and $A^{H}=A^{\text {co } H^{*}}$. The category of left $A \# H$-modules is isomorphic to ${ }_{A} \mathcal{M}^{H^{*}}$, the category of relative $\left(A, H^{*}\right)$-Hopf modules, i.e. $k$-modules together with a left $A$-action and a right $H^{*}$-coaction, satisfying an appropriate compatibility condition. Thus $[8]$ brings us necessary and sufficient conditions for a relative Hopf module to be projective as a module over the ring of coinvariants. In this paper, we will generalize these results to relative $(A, H)$-Hopf modules, where $H$ is an arbitrary Hopf algebra with bijective antipode over a commutative ring $k$, and $A$ is a ( $k$-flat) right $H$-comodule algebra.

Our main result is Theorem 2.1, where we give necessary and sufficient conditions for projectivity of a relative Hopf module over the subring of coinvariants $B=A^{\mathrm{coH}}$. The main tool - based on the methods developed in [7] and [8] - is the basic fact that the canonical structure of $A$ as left-right $(A, H)$-Hopf module is such that ${ }_{A} \operatorname{Hom}^{\mathrm{H}}(\mathrm{A}, \mathrm{A})$, the $k$-module consisting of $A$-linear and $H$-colinear maps, is isomorphic to $B$ (see Section 1). The result can be improved if we assume that there

1991 Mathematics Subject Classification. 16W30.

Key words and phrases. Relative Hopf module, coinvariants, projective object. 
exists a total integral $\phi: H \rightarrow A$, see Proposition 2.2. In Proposition 2.5, we look at relative modules that are coinvariantly generated, and we will present some conditions that are sufficient, but in general not necessary for projectivity. These conditions have the advantage that they are easier to verify than the ones from Theorem 2.1; they turn out to be necessary if the coinvariants functor is exact.

In Sections 3 and 4 , we will work over a field $k$. Our methods will be applied to discuss properties of injective and projective dimension in the category of relative Hopf modules (Section 3), and semisimplicity of the the category of relative Hopf modules. Our main result is Corollary 4.5, where we give a sufficient condition for the category of relative Hopf modules to be semisimple.

Let us finally mention that our results may be applied in the following particular cases:

- $A=H$ with comodule structure map $\Delta$. In this case, $B$ is isomorphic to $k$ as $k$-algebra and trivial $H$-comodule.

- A crossed product $A=R \#_{\sigma} H$ where $R$ is an algebra with $H$-action and $\sigma$ is an invertible map in $H_{o m}\left(H \otimes_{k} H, R\right)$ [10, Definition 7.1.1] is a right $H$-comodule algebra such that $A^{\mathrm{coH}}=$ $R$. The comodule map is given by $a \#{ }_{\sigma} h: \rightarrow a \#_{\sigma} h_{1} \otimes h_{2}$.

- $A=\oplus_{g \in G} A_{g}$ is a $G$-graded $k$-algebra for a group $G$ and $H=$ $k G$ is the group algebra of $G$. Then $A$ is a right $H$-comodule algebra, $A^{\mathrm{co} H}=A_{1}$ and ${ }_{A} \mathcal{M}^{H}$ is the category of $G$-graded left $A$-modules.

- $H=k[G]$, the affine coordinate ring of an affine $k$-group scheme $G$ and $A$ is a right $k[G]$-comodule algebra. A right $k[G]$-comodule is also called a left $G$-module (see [5], [9]). If $G$ is linearly reductive, meaning that every $G$-module is completely reducible, then the functor $(-)^{\operatorname{cok}[G]}: \mathcal{M}^{k[G]} \rightarrow{ }_{k} \mathcal{M}$ is exact. In the particular case of an affine algebraic group $G$ over an algebraically closed field $k$ acting rationally on $A,{ }_{A} \mathcal{M}^{k[G]}$ is the category of rational $(A, G)$-modules (see [6], [10]).

For more detail on Hopf algebras and the category of relative Hopf modules, we refer to the literature, see for example [1], [2], [3], [4], [1], [13.

\section{Preliminary Results}

Troughout this paper, $k$ will be a commutative ring, and $H$ will be a Hopf algebra with bijective antipode $S$. We freely use the SweedlerHeyneman notation for the comultiplication:

$$
\Delta(h)=h_{1} \otimes h_{2}
$$


We use a similar notation for the right $H$-coaction $\rho$ on a right $H$ comodule $M$ :

$$
\rho(m)=m_{0} \otimes m_{1}
$$

$\mathcal{M}^{H}$ will be the category of right $H$-comodules and right $H$-colinear maps. The $k$-module consisting of all right $H$-comodule maps between two right $H$-comodules $M$ and $N$ will be denoted by $\operatorname{Hom}^{\mathrm{H}}(\mathrm{M}, \mathrm{N})$.

The tensor product of two right $H$-comodules $M$ and $N$ is again a right $H$-comodule. The right $H$-coaction on $M \otimes N$ is given by

$$
\rho(m \otimes n)=m_{0} \otimes n_{0} \otimes m_{1} n_{1}
$$

For any right $H$-comodule $M$,

$$
M^{\mathrm{co} H}=\{m \in M \mid \rho(m)=m \otimes 1\}
$$

is called the submodule of coinvariants of $M$.

A right $H$-comodule algebra $A$ is an algebra in the category $\mathcal{M}^{H}$. This means that $A$ is a right $H$-comodule and a $k$-algebra such that the unit and multiplication maps are right $H$-colinear:

$$
\rho_{A}(a b)=a_{0} b_{0} \otimes a_{1} b_{1} \quad \text { and } \quad \rho_{A}\left(1_{A}\right)=1_{A} \otimes 1_{H}
$$

A (left-right) relative $(A, H)$-Hopf module is a $k$-module $M$ together with a left $A$-action and a right $H$-coaction such that

$$
\rho(a m)=a_{0} m_{0} \otimes a_{1} m_{1}
$$

for all $a \in A$ and $m \in M .{ }_{A} \mathcal{M}^{H}$ is the category of relative $(A, H)$ Hopf modules and left $A$-linear and right $H$-colinear maps. For two relative Hopf modules $M$ and $N$, we write ${ }_{A} \operatorname{Hom}^{\mathrm{H}}(\mathrm{M}, \mathrm{N})$ for the $k$ module consisting of all $A$-linear $H$-colinear maps from $M$ to $N$.

$B=A^{\mathrm{co} H}$ is a $k$-algebra. The coinvariants $M^{\mathrm{co} H}$ of a relative Hopf module $M$ form a $B$-module.

Lemma 1.1. (1) Take $M \in \mathcal{M}^{H}$ and $N \in{ }_{A} \mathcal{M}^{H}$.

a) $N \otimes M \in{ }_{A} \mathcal{M}^{H}$, with left $A$-action given by

$$
a(n \otimes m)=(a n) \otimes m
$$

b) If $H$ is commutative, then $M \otimes N \in{ }_{A} \mathcal{M}^{H}$, with left $A$ action given by

$$
a(m \otimes n)=m \otimes a n
$$

(2) Let $A$ be commutative, and take $M, N \in{ }_{A} \mathcal{M}^{H}$. Then $M \otimes_{A} N \in$ ${ }_{A} \mathcal{M}^{H}$, under the coaction

$$
\rho(m \otimes n)=m_{0} \otimes n_{0} \otimes m_{1} n_{1}
$$

(3) Take $M, N \in{ }_{A} \mathcal{M}^{H}$, and assume that $M$ is finitely generated projective as a left $A$-module. 
a) ${ }_{A} \operatorname{Hom}(\mathrm{M}, \mathrm{N}) \in \mathcal{M}^{\mathrm{H}}$ and

$$
{ }_{A} \operatorname{Hom}^{\mathrm{H}}(\mathrm{M}, \mathrm{N})={ }_{\mathrm{A}} \operatorname{Hom}(\mathrm{M}, \mathrm{N})^{\mathrm{coH}}
$$

b) $N \cong{ }_{A} \operatorname{Hom}(\mathrm{A}, \mathrm{N}) \in{ }_{\mathrm{A}} \mathcal{M}^{\mathrm{H}}$, with left $A$-action

$$
(a f)(u)=f(u a)
$$

c) If $A$ is commutative, then ${ }_{A} \operatorname{Hom}(\mathrm{M}, \mathrm{N}) \in{ }_{\mathrm{A}} \mathcal{M}^{\mathrm{H}}$.

Proof. 1a) The only nontrivial thing that we have to show is the compatibility of the right $H$-coaction with the left $A$-action. For any $a \in A$, $m \in M$ and $n \in N$, we have

$$
\rho_{N \otimes M}((a n) \otimes m)=a_{0} n_{0} \otimes m_{0} \otimes a_{1} n_{1} m_{1}=a_{0}(n \otimes m)_{0} \otimes a_{1}(n \otimes m)_{1}
$$

1b) As in 1a), we have to prove compatibility. For $a \in A, m \in M$ and $n \in N$, we have

$$
\begin{aligned}
& \rho_{M \otimes N}(m \otimes a n)=m_{0} \otimes a_{0} n_{0} \otimes m_{1} a_{1} n_{1} \\
& \quad=m_{0} \otimes a_{0} n_{0} \otimes a_{1} m_{1} n_{1}=a_{0}(m \otimes n)_{0} \otimes a_{1}(m \otimes n)_{1}
\end{aligned}
$$

2) The right $H$-coaction on $M \otimes_{A} N$ is well-defined since

$$
\rho(m a \otimes n)=m_{0} a_{0} \otimes n_{0} \otimes m_{1} a_{1} n_{1}=\rho(m \otimes a n)
$$

and it is straightforward to verify that $M \otimes_{A} N$ is a right $H$-comodule. Finally

$$
\rho_{M \otimes_{A} N}(a m \otimes n)=a_{0} m_{0} \otimes n_{0} \otimes a_{1} m_{1} n_{1}=a_{0}(m \otimes n)_{0} \otimes a_{1}(m \otimes n)_{1}
$$

3a) The fact that $M$ is finitely generated projective as a left $A$-module implies that we have a natural isomorphism

$$
{ }_{A} \operatorname{Hom}(\mathrm{M}, \mathrm{N}) \otimes \mathrm{H} \cong{ }_{\mathrm{A}} \operatorname{Hom}(\mathrm{M}, \mathrm{N} \otimes \mathrm{H})
$$

Using this isomorphism, we define a map

$$
\pi:{ }_{A} \operatorname{Hom}(\mathrm{M}, \mathrm{N}) \rightarrow{ }_{\mathrm{A}} \operatorname{Hom}(\mathrm{M}, \mathrm{N}) \otimes \mathrm{H} ; \quad \pi(\mathrm{f})=\mathrm{f}_{0} \otimes \mathrm{f}_{1}
$$

by

$$
(\pi(f))(m)=f_{0}(m) \otimes f_{1}=f\left(m_{0}\right)_{0} \otimes S^{-1}\left(m_{1}\right) f\left(m_{0}\right)_{1}
$$

defining a right $H$-coaction on ${ }_{A} \operatorname{Hom}(\mathrm{M}, \mathrm{N})$.

Take $f \in{ }_{A} \operatorname{Hom}^{\mathrm{H}}(\mathrm{M}, \mathrm{N})$ and $m \in M$. Then

$$
f(m)_{0} \otimes f(m)_{1}=f\left(m_{0}\right) \otimes m_{1}
$$

So

$$
\begin{aligned}
\pi(f)(m) & =f_{0}(m) \otimes f_{1}=f\left(m_{0}\right)_{0} \otimes S^{-1}\left(m_{1}\right) f\left(m_{0}\right)_{1} \\
= & f\left(m_{0}\right) \otimes S^{-1}\left(m_{2}\right) m_{1}=f\left(m_{0}\right) \otimes \varepsilon\left(m_{1}\right) 1=(f \otimes 1)(m)
\end{aligned}
$$


proving that $f$ is coinvariant. Conversely, take a coinvariant $f \in$ ${ }_{A} \operatorname{Hom}(\mathrm{M}, \mathrm{N})^{\mathrm{coH}}$. For every $m \in M$ we have

$$
f_{0}(m) \otimes f_{1}=f\left(m_{0}\right)_{0} \otimes S^{-1}\left(m_{1}\right) f\left(m_{0}\right)_{1}=f(m) \otimes 1
$$

and we deduce that

$$
\begin{aligned}
f\left(m_{0}\right) & \otimes m_{1}=f\left(m_{0}\right) \otimes m_{1} 1 \\
& =f\left(m_{0}\right)_{0} \otimes m_{2} S^{-1}\left(m_{1}\right) f\left(m_{0}\right)_{1} \\
& =f(m)_{0} \otimes f(m)_{1}
\end{aligned}
$$

so $f$ is $H$-colinear, as needed.

3b) By 3a), ${ }_{A} \operatorname{Hom}(\mathrm{A}, \mathrm{N})$ is a right $H$-comodule. The map

$$
\psi:{ }_{A} \operatorname{Hom}(\mathrm{A}, \mathrm{N}) \rightarrow \mathrm{N}, \psi(\mathrm{f})=\mathrm{f}(1)
$$

is clearly an isomorphism of $A$-modules. $\psi$ is also right $H$-colinear, since

$$
\begin{gathered}
\psi(f)_{0} \otimes \psi(f)_{1}=f(1)_{0} \otimes f(1)_{1}=f\left(1_{0}\right)_{0} \otimes S^{-1}(1) f\left(1_{0}\right)_{1} \\
=f_{0}(1) \otimes f_{1}=\psi\left(f_{0}\right) \otimes f_{1}
\end{gathered}
$$

3c) ${ }_{A} \operatorname{Hom}(\mathrm{M}, \mathrm{N})$ is an $A$-module, and, by $\left.3 \mathrm{a}\right)$, it is a right $H$-comodule. The $A$-action and $H$-coaction are compatible since

$$
\begin{aligned}
\left((a f)_{0}\right. & \left.\otimes(a f)_{1}\right)(m)=\left((a f)\left(m_{0}\right)\right)_{0} \otimes S^{-1}\left(m_{1}\right)\left((a f)\left(m_{0}\right)\right)_{1} \\
& =a_{0}\left(f\left(m_{0}\right)_{0}\right) \otimes S^{-1}\left(m_{1}\right) a_{1}\left(f\left(m_{0}\right)_{1}\right) \\
& =a_{0}\left(f\left(m_{0}\right)_{0}\right) \otimes a_{1} S^{-1}\left(m_{1}\right)\left(f\left(m_{0}\right)_{1}\right)=a_{0}\left(f_{0}(m)\right) \otimes a_{1} f_{1} \\
& =\left(a_{0} f_{0}\right)(m) \otimes a_{1} f_{1}=\left(a_{0} f_{0} \otimes a_{1} f_{1}\right)(m)
\end{aligned}
$$

and this proves that ${ }_{A} \operatorname{Hom}(\mathrm{M}, \mathrm{N}) \in{ }_{\mathrm{A}} \mathcal{M}^{\mathrm{H}}$.

Consider the functor

$$
(-)^{\mathrm{co} H}:{ }_{A} \mathcal{M}^{H} \longrightarrow{ }_{B} \mathcal{M}
$$

$(-)^{c o H}$ commutes with direct sums, and has a left adjoint

$$
T=A \otimes_{B}-:{ }_{B} \mathcal{M} \longrightarrow{ }_{A} \mathcal{M}^{H}
$$

The unit and counit of the adjunction are the following: for $N \in{ }_{B} \mathcal{M}$ and $M \in{ }_{A} \mathcal{M}^{H}$ :

$$
\begin{gathered}
u_{N}: N \longrightarrow\left(A \otimes_{B} N\right)^{\mathrm{co} H}, u_{N}(n)=1 \otimes n \\
c_{M}: A \otimes_{B} M^{\mathrm{co} H} \longrightarrow M, c_{M}(a \otimes m)=a m
\end{gathered}
$$

Lemma 1.2. (1) The functor $(-)^{\mathrm{co} H}$ is naturally isomorphic to

$$
{ }_{A} \operatorname{Hom}^{\mathrm{H}}(\mathrm{A},-):{ }_{\mathrm{A}} \mathcal{M}^{\mathrm{H}} \longrightarrow{ }_{\mathrm{B}} \mathcal{M}
$$

(2) $c_{A^{(I)}}$ is an isomorphism for any set $I$.

(3) $u_{B^{(I)}}$ is an isomorphism for any set $I$. 
Proof. 1) By Lemma 1.1 (3), ${ }_{A} \operatorname{Hom}^{\mathrm{H}}(\mathrm{A}, \mathrm{M})={ }_{\mathrm{A}} \operatorname{Hom}(\mathrm{A}, \mathrm{M})^{\mathrm{coH}}$, and ${ }_{A} \operatorname{Hom}(\mathrm{A}, \mathrm{M})$ is isomorphic to $M$ in ${ }_{A} \mathcal{M}^{H}$. So ${ }_{A} \operatorname{Hom}^{\mathrm{H}}(\mathrm{A}, \mathrm{M})$ is $B$ isomorphic to $M^{\mathrm{co} H}$.

2) It suffices to observe that

$$
\left(A^{(I)}\right)^{\mathrm{co} H}=\left(A^{\mathrm{co} H}\right)^{(I)}=B^{(I)}
$$

and

$$
c_{A^{(I)}}: A \otimes_{B}\left(A^{\mathrm{co} H}\right)^{(I)} \longrightarrow A^{(I)}
$$

is the canonical isomorphism.

3) From the fact that $u$ and $c$ are the unit and the counit of the adjunction, we derive that

$$
\left(c_{A^{(I)}}\right)^{\mathrm{co} H} \circ u_{\left(A^{(I)}\right)}{ }^{\mathrm{co} H}=1_{\left(A^{(I)}\right)^{\mathrm{co} H}}
$$

and it follows that $u_{\left(A^{(I)}\right)^{\mathrm{co} H}}=u_{\left(A^{\mathrm{co} H}\right)^{(I)}}$ is an isomorphism.

Lemma 1.3. Take $M \in{ }_{A} \mathcal{M}^{H}$. Then

(1) $M \otimes H \in{ }_{A} \mathcal{M}^{H}$ and $(M \otimes H)^{\mathrm{co} H} \cong M$ as a $B$-module.

(2) $u_{M}$ is an injection of $B$-modules.

Proof. 1) The structure maps on $M \otimes H$ are the following:

$$
a(m \otimes h)=a m \otimes h ; \rho(m \otimes h)=m_{0} \otimes h_{1} \otimes m_{1} h_{2}
$$

It is clear that the map

$$
f: M \rightarrow M \otimes H ; f(m)=m_{0} \otimes S\left(m_{1}\right)
$$

is left $B$-linear. Also

$$
\rho(f(m))=m_{0} \otimes S\left(m_{3}\right) \otimes m_{1} S\left(m_{2}\right)=m_{0} \otimes S\left(m_{1}\right) \otimes 1
$$

so $f(m) \in(M \otimes H)^{\mathrm{co} H}$, and we have a $B$-linear map

$$
f: M \rightarrow(M \otimes H)^{\mathrm{co} H}
$$

Its inverse is

$$
g:(M \otimes H)^{\mathrm{co} H} \rightarrow M ; g(m \otimes h)=\varepsilon(h) m
$$

It is easy to see that $g$ is a left inverse of $f$. Take $\sum_{i} m_{i} \otimes h_{i} \in$ $(M \otimes H)^{\mathrm{co} H}$. Then

$$
f\left(g\left(\sum_{i} m_{i} \otimes h_{i}\right)\right)=\sum_{i} \varepsilon\left(h_{i}\right) f\left(m_{i}\right)=\sum_{i} \varepsilon\left(h_{i}\right) m_{i 0} \otimes S\left(m_{i 1}\right)
$$

We also have that

$$
\sum_{i} m_{i 0} \otimes h_{i 1} \otimes m_{i 1} h_{i 2}=\sum_{i} m_{i} \otimes h_{i} \otimes 1
$$


applying $S$ to the third tensor factor, and then multiplying the second and third factor, we obtain

$$
\sum_{i} \varepsilon\left(h_{i}\right) m_{i 0} \otimes S\left(m_{i 1}\right)=\sum_{i} m_{i} \otimes h_{i}
$$

and it follows that $g$ is also a right inverse of $f$.

2) Set $W=M \otimes H$. By Lemma 1.2,

$$
\left(c_{W}\right)^{\mathrm{coH}} \circ u_{W^{c o H}}=1_{W^{c o H}}
$$

By 1$), W^{\mathrm{co} H} \cong M$ as $B$-modules, so $u_{M}$ is an injection of $B$-modules.

\section{Projectivity of Relative Hopf modules}

We keep the notation of Section 11. We will now discuss when a relative Hopf module is a projective object as a $B$-module.

Theorem 2.1. For a relative Hopf module $P \in{ }_{A} \mathcal{M}^{H}$, the following conditions are equivalent:

(1) $P$ is projective in ${ }_{B} \mathcal{M}$;

(2) $A \otimes_{B} P$ is isomorphic in ${ }_{A} \mathcal{M}^{H}$ to a direct summand of a direct sum of copies of $A$, and $u_{P}$ is surjective (bijective);

(3) There is a direct summand $M$ in ${ }_{A} \mathcal{M}^{H}$ of a direct sum of copies of $A$ such that $M^{\mathrm{coH}} \cong P$ as a $B$-module.

Proof. 1$) \Rightarrow 2$ ). Let $p: B^{(I)} \longrightarrow P$ be a split epimorphism in ${ }_{B} \mathcal{M}$. Then

$$
1 \otimes p: A \otimes_{B} B^{(I)} \longrightarrow A \otimes_{B} P
$$

is a split epimorphism in ${ }_{A} \mathcal{M}^{H}$ and $A \otimes_{B} B^{(I)} \cong A^{(I)}$ in ${ }_{A} \mathcal{M}^{H}$. Furthermore

$$
u_{P} \circ p=(1 \otimes p)^{\mathrm{co} H} \circ u_{B^{(I)}}
$$

and, by Lemma 1.2, $u_{B^{(I)}}$ is an isomorphism. Thus $u_{P}$ is an epimorphism and it follows from Lemma 1.3 that $u_{P}$ is bijective.

$2) \Rightarrow 3$ ). If $u_{P}: P \longrightarrow\left(A \otimes_{B} P\right)^{\mathrm{co} H}$ is an epimorphism (and therefore an isomorphism by Lemma 1.3) and if $M$ is a direct summand in ${ }_{A} \mathcal{M}^{H}$ of a direct sum of copies of $A$ isomorphic to $A \otimes_{B} P$, then $M$ satisfies the required condition.

$3) \Rightarrow 1$ ) Let $M$ be a direct summand in ${ }_{A} \mathcal{M}^{H}$ of a direct sum of copies of $A$, such that $M^{\mathrm{co} H} \cong P$ as a $B$-module. Then there exists a split epimorphism $f: A^{(I)} \longrightarrow M$ in ${ }_{A} \mathcal{M}^{H}$. Thus

$$
f^{\mathrm{co} H}:\left(A^{(I)}\right)^{\mathrm{co} H} \longrightarrow M^{\mathrm{co} H} \cong P
$$


is a split epimorphism in ${ }_{B} \mathcal{M}$ and $\left(A^{(I)}\right)^{\mathrm{co} H} \cong B^{(I)}$. So $P$ is projective in ${ }_{B} \mathcal{M}$.

Recall that a total integral is an $H$-colinear map $\phi: H \rightarrow A$ such that $\phi\left(1_{H}\right)=1_{A}$. If there exists a total integral, then $u_{N}$ is an isomorphism of $B$-modules, for every $N \in{ }_{B} \mathcal{M}$ (see e.g. [3, Lemma 23]). From Theorem 2.1, we easily obtain the following result:

Proposition 2.2. Assume that there exists a total integral $H \rightarrow A$, and take $P \in{ }_{A} \mathcal{M}^{H} . P$ is projective as a $B$-module if and only if $A \otimes_{B} P$ is isomorphic in ${ }_{A} \mathcal{M}^{H}$ to a direct summand of a direct sum of copies of $A$.

An $H$-ideal $I$ of $A$ is an $H$-subcomodule of $A$ which is also an ideal of $A$. We will say that $A$ is $H$-simple if $A$ has no nontrivial $H$-ideals.

Lemma 2.3. If $A$ is commutative $H$-simple, then $A^{\mathrm{co} H}$ is a field.

Proof. Let $a$ be a nonzero element in $A^{\mathrm{co} H}$. Then $A a$ is a nonzero $H$ ideal of $A$. But $A$ is $H$-simple and $A a \neq 0$, so $A a=A$. Hence, we can find an element $b$ in $A$ such that $b a=1$.

Theorem 2.1 gives necessary and sufficient conditions for the projectivity of $M \in{ }_{A} \mathcal{M}^{H}$ as a $B$-module. These conditions might be difficult to check, and this is why we look for sufficient conditions that are easier. $M \in{ }_{A} \mathcal{M}^{H}$ is called coinvariantly generated if $M=A M^{\mathrm{coH}}$.

Lemma 2.4. For any $B$-module $M, A \otimes_{B} M$ is coinvariantly generated. In particular, $A$ is coinvariantly generated.

Proof. Consider the $k$-linear map

$$
f: A\left(A \otimes_{B} M\right)^{c o H} \rightarrow A \otimes_{B} M ; f(a(u \otimes m))=a u \otimes m
$$

If $u \otimes m \in\left(A \otimes_{B} M\right)^{c o H}$, then

$$
u_{0} \otimes m \otimes u_{1}=(u \otimes m)_{0} \otimes(u \otimes m)_{1}=u \otimes m \otimes 1
$$

From this relation, we deduce that

$$
\begin{aligned}
(a u \otimes m)_{0} & \otimes(a u \otimes m)_{1}=a_{0} u_{0} \otimes m \otimes a_{1} u_{1} \\
= & a_{0} u \otimes m \otimes a_{1}=a_{0}(u \otimes m) \otimes a_{1} \\
= & \left(f \otimes i d_{H}\right)\left(a_{0}(u \otimes m)_{0} \otimes a_{1}(u \otimes m)_{1}\right)
\end{aligned}
$$

proving that $f$ is $H$-colinear. It is clear that $f$ is $A$-linear, and the $k$-linear map

$$
g: A \otimes_{B} M \rightarrow A\left(A \otimes_{B} M\right)^{\mathrm{co} H} ; \quad u \otimes m \mapsto u(1 \otimes m)
$$

is a left and right inverse of $f$. 
Proposition 2.5. Take $P \in{ }_{A} \mathcal{M}^{H}$ and consider the following conditions:

(1) $A \otimes_{B} P$ is projective in ${ }_{A} \mathcal{M}^{H}$;

(2) there exists a projective coinvariantly generated object $M \in$ ${ }_{A} \mathcal{M}^{H}$ such that $M^{c o H} \cong P$ as a $B$-module;

(3) $P$ is projective as a $B$-module.

Then 1) $\Rightarrow$ 2) $\Rightarrow$ 3). If the functor $(-)^{\mathrm{co} H}:{ }_{A} \mathcal{M}^{H} \rightarrow{ }_{B} \mathcal{M}$ is exact, then conditions 1), 2) and 3) are equivalent.

Note that the functor $(-)^{\mathrm{coH}}:{ }_{A} \mathcal{M}^{H} \rightarrow{ }_{B} \mathcal{M}$ is exact in the following situations:

- $(-)^{\mathrm{co} H}:{ }_{A} \mathcal{M}^{H} \rightarrow{ }_{k} \mathcal{M}$ is exact; this is the case if $k$ is a field and $H$ is a cosemisimple Hopf algebra [11, Lemma 2.4.3].

- $A$ is right $H$-coflat (see e.g. [3, Lemma 22]); if $k$ is a field, then this condition is equivalent to $A$ to being injective in $\mathcal{M}^{H}$ (see e.g. [3, Theorem 1]).

Proof. 1) $\Rightarrow 2$ ). Let $f: B^{(I)} \rightarrow P$ be an epimorphism in ${ }_{B} \mathcal{M}$. Then

$$
1 \otimes f: A \otimes_{B} B^{(I)} \rightarrow A \otimes_{B} P
$$

is an epimorphism in ${ }_{A} \mathcal{M}^{H}$. Therefore $1 \otimes f$ splits and we have the following commutative diagram with exact rows:

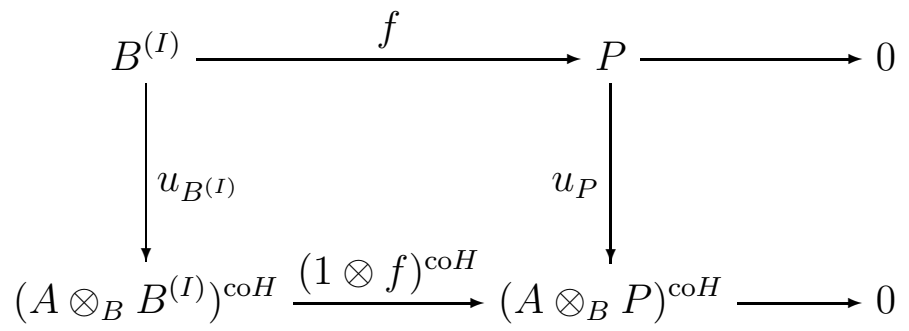

By Lemma 1.2, $u_{B^{(I)}}$ is an isomorphism. Therefore, $u_{P}$ is surjective and, by Lemma 1.3, it is an isomorphism. Thus $M=A \otimes_{B} P$ satisfies condition 2).

2) $\Rightarrow 3$ ) Consider the map

$$
f: A^{\left(M^{\mathrm{co} H}\right)} \rightarrow M ; f\left(\left(a_{m}\right)_{m \in M^{\mathrm{co} H}}\right)=\sum_{m \in M^{\mathrm{co} H}} a_{m} m
$$

For each $m \in M^{\mathrm{co} H}$, we have $\rho(m)=m \otimes 1$, hence

$$
\rho_{M}\left(\sum_{m \in M^{c o H}}\left(a_{m} m\right)\right)=\sum_{m \in M^{c o H}}\left(a_{m}\right)_{0} m \otimes\left(a_{m}\right)_{1}
$$


On the other hand

$$
\left(a_{m}\right)_{m \in M^{\mathrm{co} H}}=\sum_{m \in M^{\mathrm{co} H}}\left(0,0, \cdots, a_{m}, 0, \cdots, 0\right)
$$

The linearity of $\rho$ implies that

$$
\begin{aligned}
\left(\left(a_{m}\right)_{m \in M^{\mathrm{co} H}}\right)_{0} \otimes\left(\left(a_{m}\right)_{m \in M^{\mathrm{coH}}}\right)_{1} & \\
& =\sum_{m \in M^{\mathrm{co} H}}\left(0, \cdots 0, a_{m}, 0, \cdots 0\right)_{0} \otimes\left(0, \cdots 0, a_{m}, 0, \cdots 0\right)_{1} \\
& =\sum_{m \in M^{\mathrm{co} H}}\left(0, \cdots 0,\left(a_{m}\right)_{0}, 0, \cdots 0\right) \otimes\left(a_{m}\right)_{1}
\end{aligned}
$$

Thus

$$
\begin{aligned}
\left(f \otimes i d_{H}\right) & \left(( ( a _ { m } ) _ { m \in M ^ { \mathrm { co } H } } ) _ { 0 } \otimes \left(\left(a_{m}\right)_{\left.\left.m \in M^{\mathrm{co} H}\right)_{1}\right)}\right.\right. \\
= & \left(f \otimes i d_{H}\right)\left(\sum_{m \in M^{\mathrm{co} H}}\left(0, \cdots 0,\left(a_{m}\right)_{0}, 0, \cdots 0\right) \otimes\left(a_{m}\right)_{1}\right) \\
& =\sum\left(a_{m}\right)_{0} m \otimes\left(a_{m}\right)_{1}
\end{aligned}
$$

and it follows that $f$ is $H$-colinear. It is clear that $f$ is $A$-linear, so it is a morphism in ${ }_{A} \mathcal{M}^{H}$. $f$ is an epimorphism by the assumption $M=A M^{\text {co } H} . \quad f$ splits since $M$ is a projective object in ${ }_{A} \mathcal{M}^{H}$, so $M$ is a direct summand in ${ }_{A} \mathcal{M}^{H}$ of a direct sum of copies of $A$. By Theorem 2.1, $P$ is projective as a $B$-module.

$3) \Rightarrow 1) P$ is projective in ${ }_{B} \mathcal{M}$, so the functor $\operatorname{Hom}_{\mathrm{B}}(\mathrm{P},-):{ }_{\mathrm{B}} \mathcal{M} \rightarrow$ ${ }_{\mathrm{k}} \mathcal{M}$ is exact. On the other hand, we have an adjoint pair of functors $\left(A \otimes_{B}(-),(-)^{c o H}\right)$ and a natural isomorphism

$$
{ }_{A} \operatorname{Hom}^{\mathrm{H}}\left(\mathrm{A} \otimes_{\mathrm{B}} \mathrm{P},-\right) \cong \operatorname{Hom}_{\mathrm{B}}\left(\mathrm{P},(-)^{\mathrm{coH}}\right)
$$

By assumption, the functor $(-)^{\mathrm{co} H}$ is exact, and it follows that

$$
{ }_{A} \operatorname{Hom}^{\mathrm{H}}\left(\mathrm{A} \otimes{ }_{\mathrm{B}} \mathrm{P},-\right):{ }_{\mathrm{A}} \mathcal{M}^{\mathrm{H}} \rightarrow{ }_{\mathrm{k}} \mathcal{M}
$$

is exact, since it is isomorphic to the composition of two exact functors. This means that $A \otimes_{B} P$ is a projective object in ${ }_{A} \mathcal{M}^{H}$.

\section{Projective and injective dimension in ${ }_{A} \mathcal{M}^{H}$}

Lemma 3.1. Assume that $H$ and $A$ are commutative, and take $M, N$, $P \in{ }_{A} \mathcal{M}^{H}$, with $N$ finitely generated projective as an $A$-module.

(1) We have a $k$-isomorphism

$$
{ }_{A} \operatorname{Hom}^{\mathrm{H}}\left(\mathrm{M},{ }_{\mathrm{A}} \operatorname{Hom}(\mathrm{N}, \mathrm{P})\right) \cong{ }_{\mathrm{A}} \operatorname{Hom}^{\mathrm{H}}\left(\mathrm{M} \otimes_{\mathrm{A}} \mathrm{N}, \mathrm{P}\right)
$$

(2) The functor ${ }_{A} \operatorname{Hom}(\mathrm{N},-):{ }_{\mathrm{A}} \mathcal{M}^{\mathrm{H}} \rightarrow{ }_{\mathrm{A}} \mathcal{M}^{\mathrm{H}}$ preserves injectives. 
Proof. 1) We have a natural isomorphism

$$
\begin{gathered}
\phi:{ }_{A} \operatorname{Hom}\left(\mathrm{M},{ }_{\mathrm{A}} \operatorname{Hom}(\mathrm{N}, \mathrm{P})\right) \rightarrow{ }_{\mathrm{A}} \operatorname{Hom}\left(\mathrm{M} \otimes \otimes_{\mathrm{A}} \mathrm{N}, \mathrm{P}\right) \\
\phi(f)(m \otimes n)=f(m)(n)
\end{gathered}
$$

A standard computation shows that $f$ is $H$-colinear if and only if $\phi(f)$ is $H$-colinear.

2) Let $I$ be an injective object in ${ }_{A} \mathcal{M}^{H}$. Then the functor

$$
{ }_{A} \operatorname{Hom}^{\mathrm{H}}(-, \mathrm{I}):{ }_{\mathrm{A}} \mathcal{M}^{\mathrm{H}} \rightarrow{ }_{\mathrm{k}} \mathcal{M}
$$

is exact. $N$ is projective as an $A$-module, so $(-) \otimes_{A} N$ is also exact, and it follows from 1) that

$$
{ }_{A} \operatorname{Hom}^{\mathrm{H}}\left(-,{ }_{\mathrm{A}} \operatorname{Hom}(\mathrm{N}, \mathrm{I})\right):{ }_{\mathrm{A}} \mathcal{M}^{\mathrm{H}} \rightarrow{ }_{\mathrm{k}} \mathcal{M}
$$

is exact.

Let $k$ be a field. Then ${ }_{A} \mathcal{M}^{H}$ is a Grothendieck category with enough injective objects, and for any $M \in{ }_{A} \mathcal{M}^{H}$ we can consider the right derived functors ${ }_{A} \operatorname{Ext}^{\mathrm{H}^{i}}(M,-)$ of ${ }_{A} \operatorname{Hom}^{\mathrm{H}}(M,-):{ }_{A} \mathcal{M}^{H} \rightarrow{ }_{k} \mathcal{M}$.

Proposition 3.2. Let $k$ be a field, and assume that $H$ and $A$ are commutative. Take $M, N, P \in{ }_{A} \mathcal{M}^{H}$, with $N$ finitely generated projective as an $A$-module. Then

$$
{ }_{A} \operatorname{Ext}^{\mathrm{H}^{i}}\left(M,{ }_{A} \operatorname{Hom}(\mathrm{N}, \mathrm{P})\right) \cong{ }_{\mathrm{A}} \operatorname{Ext}^{\mathrm{H}^{\mathrm{i}}}\left(\mathrm{M} \otimes_{\mathrm{A}} \mathrm{N}, \mathrm{P}\right)
$$

Proof. By Lemma 3.1, the functors ${ }_{A} \operatorname{Hom}^{\mathrm{H}}\left(\mathrm{M},{ }_{\mathrm{A}} \operatorname{Hom}(\mathrm{N},-)\right)$ and ${ }_{A} \operatorname{Hom}^{\mathrm{H}}\left(\mathrm{M} \otimes_{\mathrm{A}} \mathrm{N},-\right)$ are isomorphic. ${ }_{A} \operatorname{Hom}(\mathrm{N},-)$ is exact, since $N$ is projective, and it preserves injectives, by Lemma 3.1. Therefore it preserves injective resolutions.

Let ${ }_{A} \operatorname{pdim}^{H}(-)$ and ${ }_{A}$ injdim ${ }^{H}(-)$ denote respectively the projective and injective dimension in ${ }_{A} \mathcal{M}^{H}$.

Corollary 3.3. With assumptions as in Proposition 3.9, we have

$$
\begin{aligned}
& { }_{A} \operatorname{pdim}^{H}\left(M \otimes_{A} N\right) \leq{ }_{A} \operatorname{pdim}^{H}(M) \\
& { }_{A} \operatorname{injdim}^{H}\left({ }_{A} \operatorname{Hom}(\mathrm{N}, \mathrm{P})\right) \leq{ }_{\mathrm{A}} \operatorname{injdim}^{\mathrm{H}}(\mathrm{P})
\end{aligned}
$$

Remarks 3.4. 1) The conclusions of Lemma 3.1, Proposition 3.2 and Corollary 3.3 remain valid without the assumption that $N$ is projective, if we assume that $A$ is semisimple.

2) As a consequence of the Fundamental Theorem for Hopf modules ([1], 1.9.4], the results of Proposition 3.2 and Corollary 3.3 also hold without the assumption that $N$ is projective, in the case where $A=H$. 


\section{Semisimplicity of the CAtegory of Relative Hopf MODULES}

Throughout this Section, $k$ will be a field, and $A$ a $k$-algebra. If $V$ is a finite dimensional vector space, then $A \otimes V$ is finitely generated projective as an $A$-module. If $V$ is an $H$-comodule, then $A \otimes V \in{ }_{A} \mathcal{M}^{H}$. So if $N \in{ }_{A} \mathcal{M}^{H}$, then, by Lemma 1.1, ${ }_{A} \operatorname{Hom}(\mathrm{A} \otimes \mathrm{V}, \mathrm{N}) \in \mathcal{M}^{\mathrm{H}}$, and ${ }_{A} \operatorname{Hom}(\mathrm{A} \otimes \mathrm{V}, \mathrm{N})$ and $\operatorname{Hom}(\mathrm{V}, \mathrm{N})$ are isomorphic as $H$-comodules.

Let $V$ be a finite-dimensional projective object of $\mathcal{M}^{H}$. Then $A \otimes V$ is a projective object of ${ }_{A} \mathcal{M}^{H}$; this follows from the fact that

$$
\begin{array}{r}
{ }_{A} \operatorname{Hom}^{\mathrm{H}}(\mathrm{A} \otimes \mathrm{V}, \mathrm{N}) \cong{ }_{\mathrm{A}} \mathrm{Hom}(\mathrm{A} \otimes \mathrm{V}, \mathrm{N})^{\mathrm{coH}} \\
\cong \operatorname{Hom}(\mathrm{V}, \mathrm{N})^{\mathrm{coH}} \cong \operatorname{Hom}^{\mathrm{H}}(\mathrm{V}, \mathrm{N})
\end{array}
$$

for all $N \in{ }_{A} \mathcal{M}^{H}$.

Proposition 4.1. $M \in{ }_{A} \mathcal{M}^{H}$ is finitely generated as an A-module if and only if there exist a finite-dimensional $H$-comodule $V$ and an epimorphism of $H$-modules and $H$-comodules $\pi: A \otimes V \longrightarrow M$.

Proof. Assume that $V$ and $\pi$ exist. Then $A \otimes V$ is finitely generated as $A$-module and $M$ is a quotient of $A \otimes V$ in ${ }_{A} \mathcal{M}$, and therefore finitely generated as a left $A$-module.

Conversely, let $M$ be finitely generated as an $A$-module by $\left\{m_{1}, \cdots, m_{n}\right\}$. For each $i$, we can find a finite dimensional $H$-subcomodule $W_{i}$ of $M$ containing $m_{i}$, see [11, 5.1.1]. $V=\sum W_{i}$ is a finite dimensional $H$ subcomodule of $M$ containing the $m_{i}$ and the $k$-linear map

$$
\pi: A \otimes V \rightarrow M ; \pi(a \otimes v)=a v
$$

is an epimorphism of $A$-modules and $H$-comodules.

Let $H^{*}$ be the linear dual of $H$, and let $M$ and $N$ be two right $H$ comodules. Then $\operatorname{Hom}_{\mathrm{k}}(\mathrm{M}, \mathrm{N})$ is a left $H^{*}$-module, under the action

$$
\left(h^{*} f\right)(m)=h^{*}\left(S^{-1}\left(m_{1}\right) f\left(m_{0}\right)_{1}\right) f\left(m_{0}\right)
$$

for all $h^{*} \in H^{*}, f \in \operatorname{Hom}_{\mathrm{k}}(\mathrm{M}, \mathrm{N})$ and $m \in M$. If $M$ and $N$ are relative Hopf modules, then ${ }_{A} \operatorname{Hom}(\mathrm{M}, \mathrm{N})$ is a left $H^{*}$-submodule of $\operatorname{Hom}_{\mathrm{k}}(\mathrm{M}, \mathrm{N})$ (see the proof of [13, Propositions 1.1 and 1.5].

A left $H^{*}$-module $M$ is called rational if the $H^{*}$-action comes from a right $H$-coaction.

Proposition 4.2. Take $M, N \in{ }_{A} \mathcal{M}^{H}$, and assume that $M$ is finitely generated as an $A$-module. Then ${ }_{A} \operatorname{Hom}(\mathrm{M}, \mathrm{N})$ is a right $H$-comodule.

Proof. Let $V$ and $\pi$ be as in Proposition 4.1. The map

$$
{ }_{A} \operatorname{Hom}_{\mathrm{A}}(\pi, \mathrm{N}):{ }_{\mathrm{A}} \operatorname{Hom}(\mathrm{M}, \mathrm{N}) \rightarrow{ }_{\mathrm{A}} \operatorname{Hom}(\mathrm{A} \otimes \mathrm{V}, \mathrm{N})
$$


is injective, and it is easy to show that it is $H^{*}$-linear. ${ }_{A} \operatorname{Hom}(\mathrm{A} \otimes \mathrm{V}, \mathrm{N})$ is an $H$-comodule, by Lemma 1.1, so it is a rational $H^{*}$-module, and - being a submodule - ${ }_{A} \operatorname{Hom}(\mathrm{M}, \mathrm{N})$ is also a rational $H^{*}$-module. The $H^{*}$-action on it is induced by an $H$-coaction, making ${ }_{A} \operatorname{Hom}(\mathrm{M}, \mathrm{N})$ a right $H$-comodule.

It follows from Proposition 4.2 that if $A$ is commutative and if $M, N \in$ ${ }_{A} \mathcal{M}^{H}$, with $M$ finitely generated as an $A$-module, then ${ }_{A} \operatorname{Hom}(\mathrm{M}, \mathrm{N}) \in$ ${ }_{\mathrm{A}} \mathcal{M}^{\mathrm{H}}$.

Recall that $M \in{ }_{A} \mathcal{M}^{H}$ is called simple if it has no proper subobjects; a direct sum of simple objects is called semisimple. ${ }_{A} \mathcal{M}^{H}$ is termed semisimple if every $M \in{ }_{A} \mathcal{M}^{H}$ is semisimple.

We say that ${ }_{A} \mathcal{M}^{H}$ satisfies condition ( $\dagger$ ) if the functor

$$
{ }_{A} \operatorname{Hom}(\mathrm{M},-):{ }_{\mathrm{A}} \mathcal{M}^{\mathrm{H}} \rightarrow \mathcal{M}^{\mathrm{H}}
$$

is exact, for every $M \in{ }_{A} \mathcal{M}^{H}$ that is finitely generated as an $A$-module. Note that ${ }_{A} \mathcal{M}^{H}$ satisfies condition ( $\dagger$ ) if $A$ is semisimple; it follows from the Fundamental Theorem for Hopf modules $([11,1.9 .4])$ that ${ }_{H} \mathcal{M}^{H}$ satisfies condition ( $\dagger$ ) if $H$ is commutative.

Proposition 4.3. Assume that ${ }_{A} \mathcal{M}^{H}$ satisfies condition $(\dagger)$, and that one of the two following conditions holds:

(1) $(-)^{\mathrm{co} H}: \mathcal{M}^{H} \rightarrow{ }_{k} \mathcal{M}$ is exact;

(2) $A$ and $H$ are commutative, and $(-)^{\mathrm{coH}}:{ }_{A} \mathcal{M}^{H} \rightarrow{ }_{B} \mathcal{M}$ is exact. If $M \in{ }_{A} \mathcal{M}^{H}$ is finitely generated as an $A$-module, then it is a projective object in ${ }_{A} \mathcal{M}^{H}$

Proof. 1) The functor ${ }_{A} \operatorname{Hom}^{\mathrm{H}}(\mathrm{M},-)$ is exact since it is the composition of the exact functors ${ }_{A} \operatorname{Hom}(\mathrm{M},-)$ and $(-)^{\mathrm{co} H}$.

2) It follows from condition $(\dagger)$ and the fact that $H$ and $A$ are commutative that

$$
{ }_{A} \operatorname{Hom}(\mathrm{M},-):{ }_{\mathrm{A}} \mathcal{M}^{\mathrm{H}} \rightarrow{ }_{\mathrm{A}} \mathcal{M}^{\mathrm{H}}
$$

is exact. Now $(-)^{\mathrm{coH}}:{ }_{A} \mathcal{M}^{H} \rightarrow{ }_{B} \mathcal{M}$ and the restriction of scalars functor ${ }_{B} \mathcal{M} \rightarrow{ }_{k} \mathcal{M}$ are both exact, and ${ }_{A} \operatorname{Hom}^{\mathrm{H}}(\mathrm{M},-)$ is again exact, being the composition of three exact functors.

Corollary 4.4. Let $A$ and $H$ be as in Proposition 4.3, and assume moreover that $A$ is left noetherian. Then every $M \in{ }_{A} \mathcal{M}^{H}$ which is finitely generated as an A-module is the direct sum of a family of simple subobjects that are finitely generated as $A$-modules, and consequently $M$ is a semisimple object in ${ }_{A} \mathcal{M}^{H}$.

Proof. Let $N$ be a subobject of $M$ in ${ }_{A} \mathcal{M}^{H}$. Then $N$ and $M / N$ are finitely generated $A$-modules, since $A$ is left noetherian. It follows from 
Proposition 4.3 that $N$ and $M / N$ are projective objects in ${ }_{A} \mathcal{M}^{H}$, and the exact sequence

$$
0 \rightarrow N \rightarrow M \rightarrow M / N \rightarrow 0
$$

splits in ${ }_{A} \mathcal{M}^{H}$.

Let $V$ be a right $H$-subcomodule of $M \in{ }_{A} \mathcal{M}^{H}$. It is clear that $A V$ is a subobject of $M$ in ${ }_{A} \mathcal{M}^{H}$.

Corollary 4.5. Let $A$ and $H$ be as in Corollary 4.4. Then ${ }_{A} \mathcal{M}^{H}$ is a semisimple category.

Proof. It is well-known (see e.g. [11, 5.1.1]) that every $m \in M$ is contained in a finite-dimensional $H$-subcomodule $V_{m}$ of $M . A V_{m}$ is then finitely generated as an $A$-module, and, by Corollary 4.4 , the direct sum in ${ }_{A} \mathcal{M}^{H}$ of a family of simple subobjects of $A V_{m}$ (and of $M)$, all finitely generated as $A$-modules. In particular, every $m \in M$ is contained in a simple subobject of $M$, and this implies that $M$ is the sum of a family of simple subobjects. Since the intersection of two simple subobjects is trivial, it follows that this sum is a direct sum.

Corollary 4.6. Let $A$ be left noetherian semisimple and $H$ cosemisimple. Then every object $M$ of ${ }_{A} \mathcal{M}^{H}$ is a direct sum in ${ }_{A} \mathcal{M}^{H}$ of a family of simple subobjects of $M$ finitely generated as A-modules. Hence $M$ is a semisimple object in ${ }_{A} \mathcal{M}^{H}$ and ${ }_{A} \mathcal{M}^{H}$ is a semisimple category.

Corollary 4.7. Let $H$ be left noetherian semisimple and cosemisimple or commutative noetherian, then every object $M$ of ${ }_{H} \mathcal{M}^{H}$ is a direct sum in ${ }_{H} \mathcal{M}^{H}$ of a family of simple subobjects of $M$ finitely generated as $H$-modules. Hence $M$ is a semisimple object in ${ }_{H} \mathcal{M}^{H}$ and ${ }_{H} \mathcal{M}^{H}$ is a semisimple category.

\section{REFERENCES}

[1] E. Abe, "Hopf Algebras", Cambridge University Press, Cambridge, 1977.

[2] S. Caenepeel, "Brauer groups, Hopf algebras and Galois theory", KMonographs Math. 4, Kluwer Academic Publishers, Dordrecht, 1998.

[3] S. Caenepeel, G. Militaru, and Shenglin Zhu, "Frobenius and separable functors for generalized module categories and nonlinear equations", Lecture Notes in Math. 1787, Springer Verlag, Berlin, 2002.

[4] S. Dăscălescu, C. Nǎstǎsescu and Ş. Raianu, "Hopf algebras: an Introduction", Monographs Textbooks in Pure Appl. Math. 235, Marcel Dekker, New York, 2001.

[5] S. Donkin, On projective modules for algebraic groups, J. London Math. Soc. 54 (1996), 75-88.

[6] I. Doraiswamy, Projectivity of modules over rings with suitable group action, Comm. Algebra 10 (1982), 787-795. 
[7] J. J. García, A. Del Río, On flatness and projectivity of a ring as a module over a fixed subring, Math. Scandin. 76 (1995), 179-192.

[8] T. Guédénon, Projectivity and flatness of a module over the subring of invariants, Comm. Algebra 29 (2001), 4357-4376.

[9] J. C. Jantzen, "Representations of algebraic groups", Pure Appl. Math. 131, Academic Press, Boston, 1987.

[10] A. R. Magid, Picard group of rings of invariants, J. Pure Appl. Algebra 17 (1980), 305-311.

[11] S. Montgomery, "Hopf algebras and their actions on rings", American Mathematical Society, Providence, 1993.

[12] D. Stefan, F. Van Oystaeyen, The Wedderburn-Malcev Theorem for comodule algebras, Comm. Algebra 27 (1999), 3569-3581.

[13] M. E. Sweedler, "Hopf algebras", Benjamin, New York, 1969.

Faculty of Applied Sciences, Vrije Universiteit Brussel, VUB, B-1050

Brussels, Belgium

E-mail address: scaenepe@vub.ac.be

URL: http://homepages.vub.ac.be/ scaenepe/

Faculty of Applied Sciences, VRije Universiteit Brussel, VUB, B-1050

Brussels, Belgium

E-mail address: guedenon@caramail.com 